\title{
DIVERSIDADE DE RAIAS MARINHAS NA COSTA DO BRASIL E SEUS ESTADOS DE AMEACGA NAGIONAL E GLOBAL
}

\author{
Diversity of seawater stingray in the coast of Brazil \\ and its status of national and global threat
}

\author{
Cicero Diogo Lins de Oliveira ${ }^{1 *}$, Carlos Yure Barbosa de Oliveira ${ }^{2}$, \\ Heitor Rodrigues Silva ${ }^{3}$, Tainá Guimarães Julio ${ }^{4}$
}

\begin{abstract}
${ }_{1}^{1}$ Doutorando do Programa de Pós-Graduação em Diversidade Biológica e Conservação nos Trópicos, na Universidade Federal de Alagoas, bolsista Capes, Instituto de Ciências Biológicas e da Saúde (ICBS), Laboratório de Conservação e Manejo de Recursos Renováveis (Lacom), Maceió - AL, Brasil. E-mail: linsdiogoc@gmail.com

${ }^{2}$ Mestrando do Programa de Pós-Graduação em Aquicultura, na Universidade Federal de Santa Catarina, bolsista Capes, Laboratório de Cultivo de Alga (LCA), Florianópolis - SC, Brasil. E-mail: yureboliveira@gmail.com

${ }^{3}$ Graduando em Ciências Biológicas na Universidade Federal de Pernambuco, Laboratório de Dinâmica de Populações Marinhas da UFRPE, Recife - PE, Brasil. E-mail: heitorrodrigues50@hotmail.com

${ }^{4}$ Doutorando do Programa de Pós-Graduação em Oceanografia, na Universidade Federal de Pernambuco, bolsista Capes, Laboratório de Compostos Orgânicos em Ecossistemas Costeiros e Marinhos (OrganoMAR), Recife - PE, Brasil. E-mail: taina.guimaraes@hotmail.com
\end{abstract}

\section{RESUMO}

O Brasil apresenta alta diversidade de peixes marinhos composta por aproximadamente 1.300 espécies. Entre essas, destacam-se as raias marinhas, por serem animais k-estrategista e possuírem grande vulnerabilidade à pesca. Nesse contexto, o presente estudo objetivou fazer o levantamento de espécies de raias que ocorrem no litoral brasileiro e seu estado de ameaça constatado pela International Union for Conservation of Nature (IUCN) e pelo Instituto Chico Mendes de Conservação da Biodiversidade (ICMBio). Para a realização do estudo, foram feitas revisões bibliográficas relacionadas à ocorrência das raias no litoral do Brasil, posteriormente, foram consultados os estados de ameaça global nas fichas da IUCN e do ICMBio de 2016, as quais foram comparadas. Foram constatadas 66 espécies, distribuídas em 35 gêneros e 11 famílias, destacando-se os gêneros Dipturus e Psammobatis com 4 espécies cada e Mobula, com 5. Das 66 espécies ocorrentes no litoral do Brasil, a IUCN já avaliou 60, sendo 40\% dessas pertencentes à categoria de Dados Insuficientes (DD), 11,7\% como Menos Preocupante (LC), 13,3\% em Próximo de Ameaça (NT), 25\% em Vulnerável (VU), 3,3\% Em Perigo (EN) e 6,7\% como Criticamente em Perigo (CR). Na ava-

Recebido em: 21/07/2018

Aprovado em: 05/09/2019

Publicado online em: $1^{\circ} . / 11 / 2019$ 
liação nacional também foram avaliadas 60 das espécies do presente estudo, sendo 46,7\% como DD, 11,7\% como LC, 3,3\% sendo NT, 16,7\% foram categorizadas como VU, 8,3\% em EN e 13,3\% em CR. Ao comparar os estados de ameaças global e nacional, 39,4\% coincidem; entretanto, 60,6\% possuem divergências na classificação de ameaça. Portanto, é notório que a atividade pesqueira tem causado sérios danos às populações de raias do litoral do Brasil e do mundo, podendo concluir que, de acordo com dados da IUCN, 35\% das espécies que ocorrem no Brasil estão em estado preocupante, já pelo ICMBio esse percentual sobe para $38,3 \%$.

Palavras-chave: conservação, elasmobrânquios, litoral brasileiro, check-list.

\section{ABSTRACT}

Brazil has a high diversity of marine fishes, consisting of approximately 1,300 species. Among these, the marine stingrays stand out because they are $k$-strategist animals and have great vulnerability to fishing. In this context, the present study aimed to survey the species of stingray that occur on the Brazilian coast and its threatened status by the International Union for Conservation of Nature (IUCN) and the Instituto Chico Mendes de Conservação da Biodiversidade (ICMBio). For the study, bibliographic reviews were made related to the occurrence of stingrays in the Brazilian coast. Later, the global threat states were consulted in the IUCN and national datasheets in the ICMBio of 2016, which were compared. A total of 66 species were found, distributed in 35 genera and 11 families, with the genera Dipturus and Psammobatis with 4 species each and Mobula with 5. Of the 66 species occurring in the Brazilian coast, the IUCN has already evaluated 60, $40.0 \%$ of which are in the Deficient Data (DD), $11.7 \%$ as Least Concern (LC), $13.3 \%$ in Near Threat (NT), 25.0\% in Vulnerable (VU), 3.3\% Endangered (EN) and 6.7\% as Critically Endangered (CR). In the national evaluation 60 species of the present study were also evaluated, $46.7 \%$ as DD, $11.7 \%$ as LC, 3.3\% being NT, $16.7 \%$ were categorized with $V U, 8.3 \%$ in EN and $13.3 \%$ in CR. When comparing global and national threat states, $39.4 \%$ agree, however, $60.6 \%$ have divergences in threat classification. Therefore, it is well known that the fishing activity has been causing serious damage to the stingray populations of the coast of Brazil and the world, and can conclude that according to IUCN data 35\% of the species occurring in Brazil are in a worrisome state, percentage increase to $38.3 \%$.

Keywords: conservation, elasmobranchs, Brazilian coast, check-list.

\section{INTRODUÇÃO}

O grupo elasmobrânquio é constituído por tubarões e raias que se distribuem em todos os mares e oceanos, em águas tropicais, subtropicais, temperadas e frias, sendo que a maioria desses indivíduos vivem no ambiente marinho e utilizam regiões estuarinas como áreas de berçário (Compagno, 1984; Castro, 1987). Além disso, os elasmobrânquios são considerados os animais mais ameaçados de extinção, com populações que alcançam declínios de até 90\% em algumas regiões (Dent \& Clarke, 2015).

Estima-se que aproximadamente $25 \%$ das espécies de tubarões e raias estejam enfrentando algum nível de ameaça (Dulvy et al., 2008). Devido às características biológicas 
como maturação tardia, baixa fecundidade e alta longevidade (Hoenig \& Gruber, 1990), eles são considerados vulneráveis às atividades humanas, tais como degradação do habitat, poluição e, principalmente, a sobre-exploração pesqueira (Dulvy et al., 2008). Atualmente, cerca de cem milhões de tubarões são mortos anualmente e a quantidade de raias ainda não é conhecida (Worm et al., 2013).

A maioria dos países que sobre-exploram as populações de tubarões possuem planos de manejos; já em relação as raias, devido à baixa quantidade de dados, existem poucos ou nenhum plano de manejo para seus recursos (Dent \& Clarke, 2015). Já os países que possuem regulamentação sobre a exploração tendem a diferir de acordo com a localização, uma vez que elas podem exibir comportamento migratório, dificultando os planos de manejo para a maioria das espécies (Compagno, 2001; Hammerschlag et al., 2011). Portanto, melhorias nas estatísticas de capturas das raias são necessárias para permitir um melhor monitoramento das populações e estratégias de manejo efetivas (Oliver et al., 2015).

Nesse contexto, o presente estudo teve como objetivo realizar o levantamento atual das espécies de raias marinhas que possuem ocorrência no litoral do Brasil, a partir da compilação de dados disponíveis e acessíveis, bem como analisar o estado de ameaça de extinção em âmbito nacional e global, com a finalidade de gerar informações básicas sobre os aspectos relacionados à diversidade e espécies prioritárias para conservação e manejo.

\section{MATERIAL E MÉTODOS}

A pesquisa baseou-se em uma revisão sistemática a qual trata-se de um tipo de investigação que visa identificar, selecionar, avaliar e sintetizar as evidências relevantes disponíveis (Galvão \& Pereira, 2014). Portanto, para o estudo, foram realizadas pesquisas de artigos e livros sobre ocorrência e diversidade de raias na costa brasileira. Posterior, as espécies foram separadas por local de ocorrência, sendo: litoral norte (rio Oiapoque, no Amapá, à foz do rio Parnaíba, no Piauí), litoral nordeste (foz do rio Parnaíba ao Recôncavo Baiano, na Bahia, incluindo Atol das Rocas e Fernando de Noronha) e litoral sudeste-sul (litoral do estado de São Paulo ao do Rio Grande do Sul), e também comparada a diversidade de cada litoral por qui-quadrado, com nível de significância de 5\% (Zar, 2013).

Foram consultadas as fixas de estados de ameaças de cada espécie na IUCN e no ICMBio de 2016, que classificam as espécies em: Não Avaliada (NE), Dados Insuficientes (DD), Menos Preocupante (LC), Próximo de Ameaça (NT), Vulnerável (VU), Em Perigo (EN), Criticamente em Perigo (CR) e Extinta (EX) (IUCN, 2014). Além disso, foi mensurado o Coeficiente de Similaridade (CS) entre as categorias nacional e internacional de cada espécie, sendo que para calcular esse coeficiente foi levado em consideração a distância entre cada categoria, com mínimo de zero (igualdade entre as categorias) e máximo 5 (maior nível de discrepância entre as categorias nacional e global), conforme a figura 1.

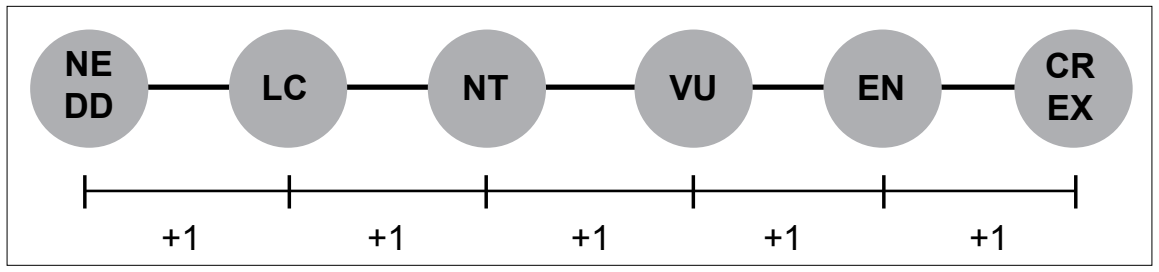

Figura 1 - Esquema de atribuição de ponto entre cada estado de ameaça Nota: $\mathrm{NE}=$ Não Avaliada; DD = Dados Insuficientes; $\mathrm{LC}=$ Menos Preocupante; NT = Próximo de Ameaça; VU = Vulnerável; EN = Em Perigo; $C R=$ Criticamente em Perigo; EX = Extinta. 
As espécies que totalizaram o coeficiente de similaridade igual ou superior a 3, entre as categorias do ICMBio e da IUCN, foram enfatizadas no presente estudo, visto que elas apresentaram categorias muito distintas entre a nacional e o global, podendo dificultar o real estado de ameaça da espécie.

\section{RESULTADOS}

Foram constatadas 11 famílias de raias marinhas (Dasyatidae, Gymnuridae, Mobulidae, Myliobatidae, Narcinidae, Pristidae, Rajidae, Rhinobatidae, Rhinopteridae, Torpedinidae e Urolophidae), totalizando 66 espécies, distribuídas em 35 gêneros, destacando-se os gêneros Dipturus e Psammobatis com 4 espécies cada e Mobula, com 5 (Tabela I).

Tabela I - Espécies de raias marinhas que ocorrem no litoral brasileiro, alguns estudos que ressaltam essa ocorrência e Coeficiente de Similaridade (CS) entre os estados de ameaça nacional e internacional

\begin{tabular}{|c|c|c|c|c|}
\hline Espécie & Estudos & $\begin{array}{c}\text { ICMBio } \\
(2016)\end{array}$ & IUCN & CS \\
\hline \multicolumn{5}{|l|}{ Dasyatidae } \\
\hline $\begin{array}{l}\text { Bathytoshia centroura } \\
\text { (Mitchill, 1815) }\end{array}$ & Gomes et al., 2010; Menezes, 2011. & $\mathrm{CR}$ & $\mathrm{LC}$ & 4 \\
\hline $\begin{array}{c}\text { Dasyatis hypostigma } \\
\text { (Santos \& Carvalho, 2004) }\end{array}$ & Nunan \& Senna, 2007; Tomás et al., 2010. & DD & $\mathrm{DD}$ & 0 \\
\hline $\begin{array}{l}\text { Dasyatis say } \\
\text { (Lesueur, 1817) }\end{array}$ & Nunes et al., 2005; Menezes, 2011; & $\mathrm{DD}$ & $\mathrm{LC}$ & 1 \\
\hline $\begin{array}{c}\text { Fontitrygon colarensis } \\
\text { (Santos, Gomes \& Charvet-Almeida, 2004) }\end{array}$ & Santos et al., 2004; Basílio et al. 2008. & VU & VU & 0 \\
\hline $\begin{array}{l}\text { Fontitrygon geijskesi } \\
\text { (Boeseman, 1948) }\end{array}$ & Nunes et al., 2005; Queiroz et al., 2008. & DD & NT & 2 \\
\hline $\begin{array}{l}\text { Himantura schmardae } \\
\quad \text { (Werner, 1904) }\end{array}$ & Carvalho et al., 2016 & DD & DD & 0 \\
\hline $\begin{array}{c}\text { Hypanus americanus } \\
\text { (Hildebrand \& Schroeder, 1928) }\end{array}$ & $\begin{array}{l}\text { Dellias et al., 2004; Silva et al., 2007; Menezes, } \\
2011 .\end{array}$ & DD & DD & 0 \\
\hline $\begin{array}{l}\text { Hypanus guttatus } \\
\text { (Bloch \& Schneider, 1801) }\end{array}$ & $\begin{array}{l}\text { Dellias et al., 2004; Silva et al., 2007. Menezes, } \\
2011 .\end{array}$ & $\mathrm{LC}$ & DD & 1 \\
\hline $\begin{array}{c}\text { Hypanus marianae } \\
\text { (Gomes, Rosa \& Gadig, 2000) }\end{array}$ & Last et al., 2016; Martins et al., 2018. & DD & DD & 0 \\
\hline $\begin{array}{l}\text { Pteroplatytrygon violácea } \\
\quad \text { (Bonaparte, 1832) }\end{array}$ & Nunan \& Senna, 2007; Mazzoleni et al., 2010. & DD & $\mathrm{LC}$ & 1 \\
\hline \multicolumn{5}{|l|}{ Gymnuridae } \\
\hline $\begin{array}{l}\text { Gymnura altavela } \\
\text { (Linnaeus, 1758) }\end{array}$ & $\begin{array}{c}\text { Nunan \& Senna, 2007; Tomás et al., 2010; } \\
\text { Menezes, } 2011 .\end{array}$ & $\mathrm{CR}$ & VU & 2 \\
\hline $\begin{array}{c}\text { Gymnura micrura } \\
\text { (Bloch \& Schneider, 1801) }\end{array}$ & $\begin{array}{l}\text { Nunes et al., 2005; Menezes, 2011; Lessa et al., } \\
2016 .\end{array}$ & NT & $\mathrm{DD}$ & 2 \\
\hline \multicolumn{5}{|l|}{ Mobulidae } \\
\hline $\begin{array}{l}\text { Manta birostris } \\
\text { (Walbaum, 1792) }\end{array}$ & Queiroz et al. 2008; Menezes, 2011. & VU & VU & 0 \\
\hline $\begin{array}{l}\text { Mobula hypostoma } \\
\text { (Bancroft, 1831) }\end{array}$ & $\begin{array}{l}\text { Nunes et al., 2005; Nunan \& Senna, 2007; } \\
\text { Menezes, } 2011 .\end{array}$ & VU & DD & 3 \\
\hline $\begin{array}{c}\text { Mobula japanica } \\
\text { (Müller \& Henle, 1841) }\end{array}$ & Nunan \& Senna, 2007; Menezes, 2011. & VU & NT & 1 \\
\hline $\begin{array}{l}\text { Mobula rochebrunei } \\
\text { (Vaillant, 1879) }\end{array}$ & Nunan \& Senna, 2007; Menezes, 2011. & VU & VU & 0 \\
\hline $\begin{array}{l}\text { Mobula tarapacana } \\
\text { (Philippi, 1892) }\end{array}$ & Nunan \& Senna, 2007; Menezes, 2011. & VU & VU & 0 \\
\hline
\end{tabular}




\begin{tabular}{|c|c|c|c|c|}
\hline Espécie & Estudos & $\begin{array}{c}\text { ICMBio } \\
(2016)\end{array}$ & IUCN & CS \\
\hline $\begin{array}{l}\text { Mobula thurstoni } \\
\text { (Lloyd, 1908) }\end{array}$ & Nunan \& Senna, 2007; Menezes, 2011. & VU & NT & 1 \\
\hline \multicolumn{5}{|l|}{ Myliobatidae } \\
\hline $\begin{array}{l}\text { Aetobatus narinari } \\
\text { (Euphrasen, 1790) }\end{array}$ & $\begin{array}{l}\text { Dellias et al. 2004; Nunes et al., 2005; Lessa et al., } \\
\text { 2016; }\end{array}$ & DD & NT & 2 \\
\hline $\begin{array}{l}\text { Myliobatis freminvillei } \\
\quad \text { (Lesueur, 1824) }\end{array}$ & Nunan \& Senna, 2007; Menezes, 2011. & EN & - & 4 \\
\hline $\begin{array}{l}\text { Myliobatis goodei } \\
\text { (Garman, 1885) }\end{array}$ & Nunan \& Senna, 2007; Menezes, 2011. & CR & DD & 5 \\
\hline $\begin{array}{c}\text { Myliobatis ridens } \\
\text { (Ruocco, Lucifora, Díaz de Astarloa, } \\
\text { Mabragaña \& Delpiani, 2012) }\end{array}$ & Rezende et al., 2015 & $\mathrm{CR}$ & - & 5 \\
\hline \multicolumn{5}{|l|}{ Narcinidae } \\
\hline $\begin{array}{c}\text { Benthobatis kreffti } \\
\text { (Rincón, Stehmann \& Vooren, 2001) }\end{array}$ & Menezes, 2011. Rincon et al., 2017. & DD & VU & 3 \\
\hline $\begin{array}{c}\text { Diplobatis pictus } \\
\text { (Palmer, 1950) }\end{array}$ & Menni \& Stehmann, 2000. & DD & VU & 3 \\
\hline Discopyge tschudii (Heckel, 1846) & Cortés et al., 2011. & DD & NT & 2 \\
\hline $\begin{array}{l}\text { Narcine bancroftii } \\
\text { (Griffith \& Smith, 1834) }\end{array}$ & Lessa et al., 2016. & DD & $\mathrm{CR}$ & 5 \\
\hline $\begin{array}{l}\text { Narcine brasiliensis } \\
\quad \text { (Olfers, } 1831)\end{array}$ & Nunes et al., 2005; Menezes, 2011. & DD & DD & 0 \\
\hline \multicolumn{5}{|l|}{ Pristidae } \\
\hline $\begin{array}{l}\text { Pristis pectinata } \\
\text { (Latham, 1794) }\end{array}$ & Gomes et al., 2010; Menezes, 2011. & CR & $\mathrm{CR}$ & 0 \\
\hline $\begin{array}{c}\text { Pristis pristis } \\
\text { (Linnaeus, } 1758)\end{array}$ & Gomes et al., 2010; Menezes, 2011. & $\mathrm{CR}$ & $\mathrm{CR}$ & 0 \\
\hline \multicolumn{5}{|l|}{ Rajidae } \\
\hline $\begin{array}{l}\text { Amblyraja frerichsi } \\
\text { (Krefft, 1968) }\end{array}$ & Bustamante et al., 2012; Rincon et al., 2017. & DD & DD & 0 \\
\hline $\begin{array}{l}\text { Atlantoraja castelnaui } \\
\text { (Miranda Ribeiro, 1907) }\end{array}$ & Gomes et al., 2010; Menezes, 2011. & EN & EN & 0 \\
\hline $\begin{array}{l}\text { Atlantoraja cyclophora } \\
\quad \text { (Regan, 1903) }\end{array}$ & Gomes et al., 2010; Menezes, 2011. & NT & VU & 1 \\
\hline $\begin{array}{l}\text { Atlantoraja platana } \\
\text { (Günther, 1880) }\end{array}$ & Gomes et al., 2010; Mazzoleni \& Schwingel, 2010. & DD & VU & 3 \\
\hline $\begin{array}{l}\text { Bathyraja brachyurops } \\
\quad \text { (Fowler, 1910) }\end{array}$ & Menni \& Stehmann, 2000; Rincon et al., 2017. & DD & $\mathrm{LC}$ & 1 \\
\hline $\begin{array}{l}\text { Bathyraja schroederi } \\
\quad \text { (Krefft, 1968) }\end{array}$ & Gomes et al., 2010; Menezes, 2011. & DD & DD & 0 \\
\hline $\begin{array}{c}\text { Breviraja spinosa } \\
\text { (Bigelow \& Schroeder, 1950) }\end{array}$ & Rincon et al., 2017 & LC & DD & 1 \\
\hline $\begin{array}{c}\text { Breviraja nigriventralis } \\
\text { (McEachran \& Matheson, 1985) }\end{array}$ & Rincon et al., 2017 & LC & DD & 1 \\
\hline $\begin{array}{c}\text { Cruriraja rugosa } \\
\text { (Bigelow \& Schroeder, 1958) }\end{array}$ & Rincon et al., 2017 & DD & DD & 0 \\
\hline $\begin{array}{c}\text { Dactylobatus clarkii (Bigelow \& Schroeder, } \\
1958 \text { ) }\end{array}$ & Soto et al., 2004; Rincon et al., 2017 & DD & DD & 0 \\
\hline $\begin{array}{l}\text { Dipturus flavirostris } \\
\text { (Philippi, 1892) }\end{array}$ & Knoff et al., 2001; Kine et al., 2007. & - & DD & 0 \\
\hline $\begin{array}{c}\text { Dipturus leptocauda } \\
\text { (Krefft \& Stehmann, 1975) }\end{array}$ & Gomes \& Costa, 2003. & DD & - & 0 \\
\hline $\begin{array}{c}\text { Dipturus menni } \\
\text { (Gomes \& Paragó, 2001) }\end{array}$ & Gomes et al., 2010; Menezes, 2011. & - & - & 0 \\
\hline
\end{tabular}




\begin{tabular}{|c|c|c|c|c|}
\hline Espécie & Estudos & $\begin{array}{c}\text { ICMBio } \\
(2016)\end{array}$ & IUCN & CS \\
\hline $\begin{array}{c}\text { Dipturus teevani } \\
\text { (Bigelow \& Schroeder, 1951) }\end{array}$ & Rincon et al., 2017. & $\mathrm{LC}$ & DD & 1 \\
\hline $\begin{array}{c}\text { Gurgesiella atlântica (Bigelow \& Schroeder, } \\
\text { 1962) }\end{array}$ & Rincon et al., 2017. & $\mathrm{LC}$ & DD & 1 \\
\hline $\begin{array}{c}\text { Gurgesiella dorsalifera } \\
\text { (McEachran \& Compagno, 1980) }\end{array}$ & Menezes, 2011; Rincon et al., 2017. & $\mathrm{LC}$ & VU & 2 \\
\hline $\begin{array}{c}\text { Malacoraja obscura } \\
\text { (Carvalho, Gomes \& Gadig, } 2005\end{array}$ & Gomes et al., 2010; Rincon et al., 2017. & $\mathrm{LC}$ & DD & 1 \\
\hline $\begin{array}{l}\text { Psammobatis bergi } \\
\quad \text { (Marini, 1932) }\end{array}$ & Mazzoleni \& Schwingel, 2010; Menezes, 2011. & DD & $\mathrm{LC}$ & 1 \\
\hline $\begin{array}{l}\text { Psammobatis extenta } \\
\quad \text { (Garman, 1913) }\end{array}$ & Gomes et al., 2010; Menezes, 2011. & DD & $\mathrm{LC}$ & 1 \\
\hline Psammobatis lentiginosa (McEachran, 1983) & Menezes, 2011. & DD & $\mathrm{DD}$ & 0 \\
\hline $\begin{array}{l}\text { Psammobatis rutrum } \\
\quad \text { (Jordan, 1891) }\end{array}$ & Gomes et al., 2010; Menezes, 2011. & DD & DD & 0 \\
\hline Rajella fuliginea & Rincon et al., 2017 & - & $\mathrm{LC}$ & 1 \\
\hline $\begin{array}{c}\text { Rajella purpuroventralis } \\
\text { (Bigelow \& Schroeder, 1962) }\end{array}$ & Rincon et al., 2017 & - & - & 0 \\
\hline $\begin{array}{c}\text { Rajella sadowskii } \\
\text { (Krefft \& Stehmann, 1974) }\end{array}$ & Gomes et al., 2010; Menezes, 2011. & DD & DD & 0 \\
\hline $\begin{array}{c}\text { Rioraja agassizi } \\
\text { (Müller \& Henle, } 1841 \text { ) }\end{array}$ & Gomes et al., 2010; Menezes, 2011. & EN & VU & 1 \\
\hline $\begin{array}{l}\text { Sympterygia acuta } \\
\text { (Garman, 1877) }\end{array}$ & Gomes et al., 2010; Menezes, 2011. & EN & VU & 1 \\
\hline $\begin{array}{l}\text { Sympterygia bonapartii } \\
\text { (Müller \& Henle, 1841) }\end{array}$ & Bornatowski et al., 2009; Gomes et al., 2010. & EN & DD & 4 \\
\hline $\begin{array}{l}\text { Zearaja chilensis } \\
\text { (Guichenot, 1848) }\end{array}$ & Rincon et al., 2017. & - & VU & 3 \\
\hline \multicolumn{5}{|l|}{ Rhinobatidae } \\
\hline $\begin{array}{c}\text { Pseudobatos horkelii } \\
\text { (Müller \& Henle, 1841) }\end{array}$ & Gomes et al., 2010; Menezes, 2011. & $\mathrm{CR}$ & $\mathrm{CR}$ & 0 \\
\hline $\begin{array}{l}\text { Pseudobatos lentiginosus } \\
\quad \text { (Garman, 1880) }\end{array}$ & Queiroz et al., 2008. & VU & NT & 1 \\
\hline $\begin{array}{l}\text { Pseudobatos percellens } \\
\quad \text { (Walbaum, 1792) }\end{array}$ & $\begin{array}{l}\text { Nunes et al., 2005; Menezes, 2011; Lessa et al., } \\
2016 .\end{array}$ & DD & NT & 2 \\
\hline $\begin{array}{l}\text { Zapteryx brevirostris } \\
\text { (Müller \& Henle, 1841) }\end{array}$ & Gomes et al., 2010; Menezes, 2011. & VU & VU & 0 \\
\hline \multicolumn{5}{|l|}{ Rhinopteridae } \\
\hline $\begin{array}{l}\text { Rhinoptera bonasus } \\
\text { (Mitchill, 1815) }\end{array}$ & $\begin{array}{l}\text { Nunes et al., 2005; Nunan \& Senna, 2007; } \\
\text { Menezes, } 2011 .\end{array}$ & DD & NT & 2 \\
\hline $\begin{array}{l}\text { Rhinoptera brasiliensis } \\
\text { (Müller, 1836) }\end{array}$ & Nunan \& Senna, 2007; Menezes, 2011. & CR & EN & 1 \\
\hline \multicolumn{5}{|l|}{ Torpedinidae } \\
\hline $\begin{array}{l}\text { Tetronarce nobiliana } \\
\text { (Bonaparte, 1835) }\end{array}$ & Menezes, 2011. & - & DD & 0 \\
\hline $\begin{array}{l}\text { Tetronarce puelcha } \\
\text { (Lahille, 1926) }\end{array}$ & Mazzoleni \& Schwingel, 2010; Menezes, 2011. & VU & DD & 3 \\
\hline \multicolumn{5}{|l|}{ Urotrygonidae } \\
\hline $\begin{array}{l}\text { Urotrygon microphtalmum } \\
\text { (Delsman, 1941) }\end{array}$ & Nunes et al., 2005. & DD & - & 0 \\
\hline
\end{tabular}

Em relação à diversidade por litoral, a maior diversidade ocorreu no litoral sudeste-sul, com 51 espécies, seguida pelo litoral Nordeste e, por último, o Norte (Figura 2). Entre 
a diversidade por litoral, o único que diferiu dos demais foi o Sudeste-Sul ( $\mathrm{p}$-valor $=0,044$ ), enquanto Norte e Nordeste não houve diferença significativa ( $\mathrm{p}$ - valor $=0,511$ ).

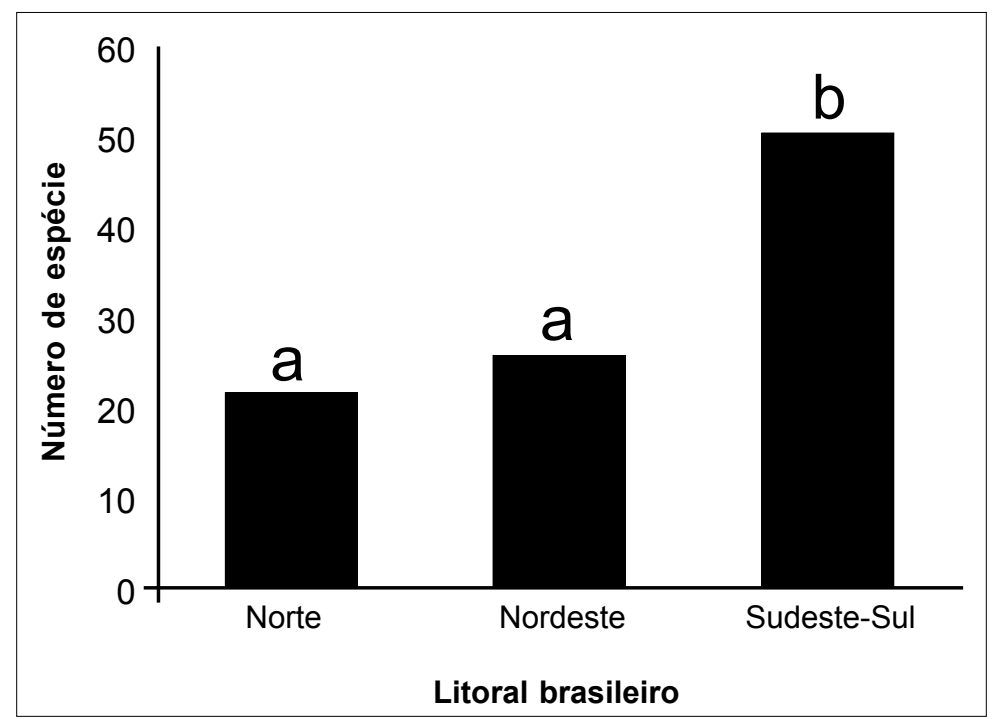

Figura 2 - Diversidade de raias marinhas por tipo de litoral do Brasil

Nota: letras iguais indicam igualdade estatística, letras diferentes mostram diferença significativa.

Das 66 espécies ocorrentes no litoral do Brasil, a IUCN já avaliou 60, sendo 40,0\% dessas pertencentes à categoria de Dados Insuficientes (DD), 11,7\% como Menos Preocupante (LC), 13,3\% em Próximo de Ameaça (NT), 25\% em Vulnerável (VU), 3,3\% Em Perigo (EN) e 6,7\% como Criticamente em Perigo. Já na avaliação nacional, quando analisada a lista vermelha do ICMBio de 2008 e 2016, o número de espécies preocupante subiu de 3 para 23. De acordo com os dados de 2016 do ICMBio, foram avaliadas 60 das espécies do presente estudo, sendo $46,7 \%$ como DD, 11,7\% como LC, 3,3\% sendo NT, 16,7\% foram categorizadas como VU, 8,3\% em EN e 13,3\% em CR. Ao comparar os estados de ameaças global e nacional, 39,4\% coincidiram, entretanto, 60,6\% possuem divergências na classificação de ameaça (Tabela I).

Das espécies que possuem divergência entre IUCN e ICMBio, 12 tiveram o Coeficiente de Similaridade superior a 2, sendo seis espécies com 3 de CS, três com 4 pontos e outras três com 5 (Tabela I).

\section{DISCUSSÃO}

Com os avanços das pesquisas, o número de espécies de raias registrado no Brasil tem aumentando. Um dos primeiros trabalhos a reunir informações de diversidade de raias marinhas no Brasil foi realizado por Fowler (1941), que, com base em citações da literatura, o autor conseguiu listar 26 raias marinhas. Em 1977, Figueiredo publicou o primeiro volume do Manual de Peixes Marinhos do Sudeste do Brasil, com destaque para os elasmobrânquios, registrando 27 espécies de raias para a região. Gadig (1998) também, ao realizar um levantamento da fauna de Chondrichthyes marinhos no estado de São Paulo, apontou 31 raias.

Lessa et al. (1999), em uma análise da diversidade de elasmobrânquios do litoral brasileiro, registraram 45 espécies de raias, além de mencionarem quatro raias que já se encontravam em processo de descrição taxonômica, porém sem exatidão a nível de espécies. Menni e Stehmann (2000) apresentaram uma lista das raias da Argentina, do Brasil e do Uruguai, sendo computada 44 espécies para o Brasil. Já Menezes et al. (2003) constataram 
54 espécies. No trabalho mais recente sobre levantamento da condrofauna, Rosa e Gadig (2014) constataram 70 espécies de raias, porém sete delas não houve a certeza em relação ao nível de espécie.

Ao analisar historicamente o número de espécies de raias marinha que ocorrem no Brasil, houve um aumento de 26 espécies de raias (Fowler, 1941) para 66 do presente estudo. Quando comparado com dados mais recente, 63 espécies eram registadas por Rosa e Gadig (2014), descartando as raias que não tinham sido identificadas até o nível de espécie, tendo um aumento de três espécies até o atual estudo. Além disso, algumas espécies tiveram mudanças de nomenclatura (Tabela II), não abortadas até então.

Tabela II - Mudanças da atual nomenclaturas das raias

\begin{tabular}{ll}
\hline Nomenclatura antiga & Nomenclatura atual \\
\hline Dasyatis americana & Hypanus amaricanus \\
Dasyatis centroura & Bathytoshia centroura \\
Dasyatis colarensis & Fontitrygon colarensis \\
Dasyatis geijskesi & Fontitrygon geijskesi \\
Dasyatis guttata & Hypanus guttata \\
Dasyatis marianae & Hypanus marianae \\
Rhinobatos horkelii & Pseudobatos horkelii \\
Rhinobatos percellens & Pseudobatos percellens \\
Rhinobatus lentiginosus & Pseudobatos lentiginosus \\
\hline
\end{tabular}

Em relação à diversidade por litoral, observa-se uma maior diversidade de raias no litoral Sudeste-Sul, que pode estar vinculado à quantidade de estudos realizados, sendo mais comum nessa região. Além disso, Menezes et al. (2003) ressaltam que nas regiões Norte e Nordeste há predominância de espécies restritas às águas tropicais, incluindo tanto espécies com distribuição restrita à porção tropical do Atlântico Sul ocidental, quanto espécies amplamente distribuídas no Atlântico ocidental tropical e subtropical. Já os litorais sudeste e sul do Brasil representam o limite meridional de ocorrência de diversas espécies predominantemente tropicais e o limite setentrional de ocorrência de espécies típicas das regiões mais temperadas do Atlântico Sul.

Entre as 66 espécies registradas neste estudo, 12 tiveram alto Coeficiente de Similaridade, mostrando grandes divergências no estado de conservação. Os motivos dessa alta diferença entre o estado de ameaça nacional e global encontram-se descritos na tabela III, podendo perceber que essas divergências estão vinculadas principalmente a área de ocorrência das espécies e à falta de informações biológicas e de pesca, que impossibilitam a avaliação do estado de conservação. Portanto, é notória a importância de mais estudos, principalmente sobre as espécies avaliadas como DD, além das espécies mencionadas com alta discrepância entre as classificações do ICMBio e da IUCN, para que se entenda o real estado de cada espécie e evite a perda de biodiversidade marinha do país.

Tabela III - Espécies de raias marinhas com altas divergências entre os estados de ameaças nacional e internacional ejustificativa Espécie Justificativa

No Brasil, a espécie encontra-se, majoritariamente, no estado do Rio Grande do Sul. Durante 1976 a 2002, a espécie apresentou reduções de 83,6\% em capturas; em outras regiões, a espécie nunca foi abundante, havendo apenas registros pontuais para a costa nordeste e central do Brasil, por isso a espécie no Brasil é categorizada como CR (ICMBio, 2016). Já de acordo com dados da IUCN, a espécie é classificada como LC por apresentar ampla distribuição, ocorrendo por todo o Atlântico, além da espécie não ser alvo de pesca no Atlântico Noroeste dos EUA acreditando-se que B. centroura na costa leste dos EUA é estável (Rosa et al., 2016). 
(continuação Tabela III)

\begin{tabular}{cl}
\hline Espécie & \multicolumn{1}{c}{ Justificativa } \\
\hline \hline Mobula hypostoma & Nacionalmente, a espécie é descrita como Vulnerável devido pertencer à família de \\
& Mobulidae, que apresenta crescente captura incidental das espécies que a compõe. \\
& Suspeita-se que o aumento das capturas incidentais pela pesca implica em uma redução \\
& populacional de no mínimo 30\% ao longo das próximas três gerações (ICMBio, 2016). Já \\
& globalmente, está na categoria DD, mesmo os dados de pesquisa de arrasto da costa leste \\
& dos Estados Unidos indiquem possíveis tendências crescentes, porém há pouca informação \\
& específica disponível sobre tendências de captura, abundância e população de todo o seu \\
& alcance (Bizzarro et al., 2009). \\
\hline & As espécies do gênero Myliobatis apresentam distribuição altamente restrita ao litoral sul do \\
& Brasil, principalmente no estado do Rio Grande do Sul, onde M. freminvillii apresentou entre \\
& os anos de 1980 a 2002 um decréscimo de 91\% da população, suspeitando de um declínio \\
& populacional de pelo menos $60 \%$, enquadrando-a como EN. Também é evidenciado alto \\
& declínio (99\%) de M. goodei entre os anos de 1974 e 2005, sugerindo assim que a redução \\
populacional ocorrida nesta área seja extensiva para a população brasileira como um todo, & o que justifica a aplicação da categoria CR. E M. ridens é considerada como espécie nova e \\
endêmica do Atlântico Sul ocidental, com registros em Santa Catarina até o norte da Argentina, \\
e a espécie, no Brasil, ocorre somente no litoral sul de Santa Catarina e Rio Grande do Sul, \\
suspeitando-se que ela tenha sofrido declínios superiores a 90\% no período que inclui três \\
gerações, qualificando a espécie como CR (ICMBio, 2016). Já globalmente, ambas as espécies \\
ou ainda não foram avaliadas ou constam como dados insuficientes, ocasionado pela falta de \\
estudos e pelas restrições de ocorrências.
\end{tabular}

Como não existem dados suficientes sobre a biologia, ecologia e dimensão dos impactos atuais e potenciais, no Brasil a espécie foi categorizada como Dados Insuficientes (ICMBio, 2016). De acordo com dados da IUCN, a espécie é classificada como vulnerável por apresentar uma Benthobatis kreffti distribuição extremamente restrita, conhecida apenas no estado de Santa Catarina, Brasil, sendo capturado em grande número pela frota de arrasto, além de que provavelmente a espécie tenha uma baixa resiliência ao esgotamento como espécie de águas profundas, com apenas um ou dois embriões por gestação (Rincon, 2004).

No Brasil, ela ocorre em águas rasas em uma área amplamente explorada pela pesca de arrasto, onde é capturada incidentalmente nas pescarias de camarão. No entanto, é uma espécie pouco conhecida, com pouca informação disponível e cujos dados relativos à biologia, à dinâmica populacional e ao estado de conservação são praticamente inexistentes, por isso é classificado Diplobatis pictus nacionalmente como DD (ICMBio, 2016). Já na IUCN, acredita-se que a espécie pode estar sub-registrada e, portanto, pescada mais intensamente do que se acredita atualmente. Apesar dessa falta de informação, esta espécie é avaliada como Vulnerável por medida de precaução, uma vez que sua distribuição coincide com a de atividade intensiva de arrasto (Carvalho \& McCord, 2006).

No Brasil, é encontrada no litoral norte, pelo menos até o Maranhão. As principais ameaças são as capturas incidentais na pesca artesanal, principalmente os arrastões de praia, e arrastos motorizados de camarão em praias e bancos rasos de lama. No entanto, o efeito desses impactos

Narcine bancroftii sobre as populações da espécie é desconhecido. Não existem dados populacionais e há pendências taxonômicas, portanto, a espécie foi avaliada como Dados Insuficientes (ICMBio, 2016). Globalmente a espécie é avaliada como Criticamente Ameaçada, com base nos declínios observados nas águas dos EUA. Além disso, acredita-se que as taxas de sobrevivência dela sejam muito baixas (Carvalho et al., 2007).

A espécie é endêmica do Atlântico Sul ocidental, ocorrendo desde o litoral do estado de São Paulo até a Argentina. No Brasil, não existem dados consistentes sobre as populações de $A$. platana e sua tendência é desconhecida, por isso, atualmente ainda não foi possível quantificar

Atlantoraja platana o declínio da espécie, sendo classificada como Dados Insuficientes (ICMBio, 2016). Já globalmente, mesmo não existindo dados de captura específicos para A. platana, ela é avaliada como Vulnerável dada a pressão realizada pela pesca, sobre outras espécies do mesmo gênero (e. g. A. castelnaui e A. cyclophora) (San Martín, 2007). 
(continuação Tabela III)

\begin{tabular}{|c|c|}
\hline Espécie & Justificativa \\
\hline Sympterygia bonapartii & $\begin{array}{l}\text { S. bonapartii é endêmica do Atlântico Sul ocidental, e a principal ameaça sobre a espécie é } \\
\text { a pesca de arrasto. A análise de dados de captura na plataforma sul do Brasil mostrou um } \\
\text { declínio de biomassa de } 94 \% \text { entre } 1980 \text { e } 2005 \text {, suspeitando um declínio populacional de pelo } \\
\text { menos } 50 \% \text {, por esse motivo, a espécie é avaliada como EN (ICMBio, 2016). Globalmente, } \\
\text { devido à falta de informação biologia, ecologia e de impacto da pesca na abundância desta } \\
\text { espécie, ela é classificada como DD, porém a espécie está se tornando cada vez mais importante } \\
\text { nas pescarias do Atlântico sudoeste (Massa \& Lamilla, 2004). }\end{array}$ \\
\hline Zearaja chilensis & $\begin{array}{l}\text { No Brasil, a espécie não foi avaliada, no entanto globalmente a espécie é dita como Vulnerável, } \\
\text { ocasionada pela sobre-exploração e ao esgotamento da população, tanto pela pressão da pesca } \\
\text { alvo quanto a capturada acidentalmente. No Chile, a biomassa total de Z. chilensis e Dipturus } \\
\text { trachydermus diminuiu em } 51 \% \text { desde } 1979 \text {, embora as estatísticas de desembarque não sejam } \\
\text { separadas por espécie. Pesquisas mostram que Z. chilensis representa } 85 \% \text { das capturas, } \\
\text { agravando o estado de conservação da Z. chilensis (Kyne et al., 2007). }\end{array}$ \\
\hline Tetronarce puelcha & $\begin{array}{l}\text { Essa espécie ocorre do sudeste do Brasil até o norte da Argentina, desde águas costeiras } \\
\text { até } 600 \mathrm{~m} \text { de profundidade. No Brasil, ela é capturada incidentalmente e descartada em } \\
\text { pescarias de arrasto sobre a plataforma continental. É uma das espécies mais sensíveis aos } \\
\text { efeitos das capturas em pesca de arrasto em Santa Catarina. Seu grande tamanho, a baixa } \\
\text { frequência, sua distribuição relativamente restrita e em manchas e as constantes operações } \\
\text { de pesca com arrasto são fatores que aumentam a vulnerabilidade da espécie, por isso ela é } \\
\text { classificada como Vulnerável (ICMBio, 2016). Já de acordo com a ficha da IUCN, a espécie é } \\
\text { classificada como DD, devido não haver informações suficientes disponíveis para avalia-la } \\
\text { (Stehmann } \text { et al., 2006). }\end{array}$ \\
\hline
\end{tabular}

\section{CONCLUSÃO}

Portanto, é notório que a atividade pesqueira tem causado sérios danos às populações de raias do litoral do Brasil e do mundo, podendo concluir que, de acordo com dados da IUCN, 35\% das espécies já avaliadas que ocorrem no Brasil estão em estado preocupante, dentro das categorias de ameaçadas de extinção; já pelo ICMBio de 2016, esse percentual sobe para $38,3 \%$. Além disso, é preciso a realização de mais estudos sobre as populações de raias, especialmente no que tange as espécies ainda não avaliadas ou as que se encontram como dados insuficientes, bem como aquelas que possuem divergência agravante entre os estados de ameaça, para que se entenda-se o real estado de cada espécie, e evite a perda da biodiversidade.

\section{REFERÊNCIAS BIBLIOGRÁFICAS}

Basílio, T.H.; Faria, V.V. \& Neto, M.A.D.A.F. Fauna de elasmobrânquios do estuário do rio Curu, Ceará, Brasil. Arquivos de Ciências do Mar, v. 41, n. 2, p. 65-72, 2008.

Bizzarro, J.; Smith, W.; Baum, J.; Domingo, A. \& Menni, R. Mobula hypostoma. The IUCN Red List of Threatened Species 2009: e.T161737A5492018. 2009. Disponível em: http://dx.doi. org/10.2305/IUCN.UK.2009-2.RLTS.T161737A5492018.en. Acesso em: 12 jul. 2018.

Bornatowski, H.; Abilhoa, V. \& Charvet-Almeida, P. Elasmobranchs of the Paraná Coast, southern Brazil, south-western Atlantic. Marine Biodiversity Records, v. 2, p. 1-6, 2009.

Bustamante, C.; Lamilla, J.; Concha, F.; Ebert, D.A. \& Bennett, M.B. Morphological characters of the thickbody skate Amblyraja frerichsi (Krefft 1968) (Rajiformes: Rajidae), with notes on its biology. PloS one, v. 7, n. 6, p. e39963, 2012. 
Carvalho, M.D.; Loboda, T.S. \& Silva, J.P.C.B. A new subfamily, Styracurinae, and new genus, Styracura, for Himantura schmardae (Werner, 1904) and Himantura pacifica (Beebe \& Tee-Van, 1941) (Chondrichthyes: Myliobatiformes). Zootaxa, v. 4175, n. 3, p. 201-221, 2016.

Carvalho, M.R. \& McCord, M.E. Diplobatis pictus. The IUCN Red List of Threatened Species 2006: e.T61404A12471946. 2006. Disponível em: http:/ / dx.doi.org/10.2305/IUCN.UK.2006. RLTS.T61404A12471946.en. Acesso em: 12 jul. 2018.

Carvalho, M.R.; McCord, M.E. \& Myers, R.A. Narcine bancroftii. The IUCN Red List of Threatened Species 2007: e.T63142A12622582. 2007. Disponível em: http://dx.doi. org/10.2305/IUCN.UK.2007.RLTS.T63142A12622582.en. Acesso em: 12 jul. 2018.

Castro J.I. The Position of Sharks in Marine Biological Comunities an Overview, in Cook, S. (Ed.). Sharks. An Inquiry into Biology, Behavior, Fisheries and Use. Proc. Of a Conf. Portland. Oregon, State University Extension Service, p. 11-17, 1987.

Compagno, L.J.V. Sharks of the World: An Annotated and Illustrated Catalogue of Shark Species Known to Date.,v. 2, n. 1, Food Agriculture Org., 278 p., 2001.

Compagno, L.J.V. Sharks of the world. Na annotated and illustrated catalogue of shark species known to date. Parts 1, FAO Species catalogue, v. 4, 655 p., 1984.

Cortés, F.; Jaureguizar, A.J.; Guerrero, A.R. \& Dogliotti, A. Influence of estuarine and continental shelf water advection on the coastal movements of apron ray Discopyge tschudii in the Southwestern Atlantic. Journal of applied ichthyology, v. 27, n. 5, p. 1278-1285, 2011.

Dellias, J.M.; Onofre, G.R.; Werneck, C.C.; Landeira-Fernandez, A.M.; Melo, F.R.; Farias, W.R. \& Silva, L.C.F. Structural composition and differential anticoagulant activities of dermatan sulfates from the skin of four species of rays, Dasyatis americana, Dasyatis gutatta, Aetobatus narinari and Potamotrygon motoro. Biochimie, v. 86, n. 9-10, p. 677-683, 2004.

Dent, F. \& Clarke, S. State of the global market for shark products. FAOFisheries and Aquaculture Technical Paper, 590 p., 2015.

Dulvy, N.K.; Baum, J.K.; Clarke, S.; Compagno, L.J.; Cortés, E.; Domingo, A.; Fordham, S.; Fowler, S.; Francis, M.P.; Gibson, C.; Martínez, J.; Musick, J.A.; Soldo, A.; Sttevens, J.D. \& Valenti, S. You can swim but you can't hide: the global status and conservation of oceanic pelagic sharks and rays. Aquatic Conservation: Marine and Freshwater Ecosystems, v. 18, n. 5, p. 459-482, 2008.

Figueiredo, J.L. Manual de peixes do Sudeste do Brasil. I. Introdução: cações, raias e quimeras. Museu de Zoologia da Universidade de São Paulo, São Paulo-SP, 111 p., 1977.

Fowler, H.W. A list of the fishes known from the coast of Brazil. Arquivos de Zoologia, v. 3, p. 115--184, 1941.

Gadig, O.B.F. Peixes cartilaginosos da costa do estado de São Paulo. Ceciliana, v. 8, p. 41-51, 1998.

Galvão, T.F. \& Pereira, M.G. Revisões sistemáticas da literatura: passos para sua elaboração. Epidemiologia e Serviços de Saúde, v. 23, p. 183-184, 2014.

Gomes, U.L. \& Costa, S.R. New records of the thintail skates Dipturus leptocauda (Rajidae, Rajinae, Rajini), with notes on its taxonomy. Biociências, v. 11, n. 1, p. 91-95, 2003.

Gomes, U.L.; Signori, C.N.; Gadig, O.B.F. \& Santos, H.R.S. Guia para identificação de tubarões e raias do Rio de Janeiro, Technical Books. 215 p., 2010. 
Hammerschlag, N.; Gallagher, A.J. \& Lazarre, D.M. A review of shark satellite tagging studies. Journal of Experimental Marine Biology and Ecology, v. 398, n. 1, p. 1-8, 2011.

Hoenig, J.M. \& Gruber, S.H. Life-history patterns in the elasmobranchs: implications or fisheries management, pages 1-16, in Pratt, H.L.; Gruber, S.H. \& Taniuchi, T. (Eds.). Elasmobranchs as living resources: advances in the biology, ecology, systematics, and the status of fisheries. NOAA Tech. Rep. 90 p., 1990.

ICMBio. Instituto Chico Mendes de Conservação da Biodiversidade. 2016. Avaliação do risco de extinção dos elasmobrânquios e quimeras no Brasil: 2010-2012. Disponível em: http:/ / www. icmbio.gov.br/cepsul/images/stories/biblioteca/download/trabalhos_tecnicos / pub_2016_avaliacao_elasmo_2010_2012.pdf.

IUCN. Guidelines for Using the IUCN Red List Categories and Criteria. Version 11. Prepared by the Standards and Petitions Subcommittee. 2014. Disponível em: https://cmsdata.iucn.org/ downloads/redlistguidelines.pdf. Acesso em: 17 jun. 2018.

Knoff, M.; São Clemente, S.C.D.; Pinto, R.M. \& Gomes, D.C. Digenea and Acanthocephala of elasmobranch fishes from the southern coast of Brazil. Memórias do Instituto Oswaldo Cruz, v. 96, n. 8, p. 1095-1101, 2001.

Kyne, P.M.; Lamilla, J.; Licandeo, R.R.; San Martín, J.M.; Stehmann, M.F.W. \& McCormack, C. Zearaja chilensis. The IUCN Red List of Threatened Species 2007: e.T63147A12623314. 2007. Disponível em: http://dx.doi.org/10.2305/IUCN.UK.2007.RLTS.T63147A12623314.en. Acesso em: 12 jul. 2018.

Last, P.R.; Carvalho, M.R.; Corrigan, S.; Naylor, G.J.; Séret, B. \& Yang, L. The Rays of the World project-an explanation of nomenclatural decisions. Rays of the World: Supplemental information. CSIRO Publishing, Melbourne, v. 40, p. 1-10, 2016.

Lessa, R.; Rodrigues, J.; Barreto, R.; Nunes, R.; Camargo, G. \& Santana, F.M. Pesca incidental de rajiformes nos arrastos de praia em Caiçara do Norte, RN. Revista Brasileira de Engenharia de Pesca, v. 8, n. 2, p. 34-41, 2016.

Lessa, R.; Santana, F.M.; Rincón, G.; Gadig, O.B.F. \& El-Deir, A.C.A. Biodiversidade de elasmobrânquios do Brasil. Recife-PE, Ministério do Meio Ambiente (MMA). 155 p., 1999.

Martins, A.P.B.; Heupel, M.R.; Chin, A. \& Simpfendorfer, C.A. Batoid nurseries: definition, use and importance. Marine Ecology Progress Series, v. 595, p. 253-267, 2018.

Massa, A. \& Lamilla, J. Sympterygia bonapartii. The IUCN Red List of Threatened Species 2004: e.T44597A10912987. 2004. Disponível em: http://dx.doi.org/10.2305/IUCN.UK.2004. RLTS.T44597A10912987.en. Acesso em: 12 jul. 2018.

Mazzoleni, R. \& Schwingel, P. Espécies de elasmobrânquios desembarcadas no porto de Itajaí, sul do Brasil. Brazilian Journal of Aquatic Science and Technology, v. 3, n. 1, p. 111-118, 2010.

Menezes, N.A. Checklist dos peixes marinhos do estado de São Paulo, Brasil. Biota Neotropica, v. 11, n. 1A, p. 33-46, 2011.

Menezes, N.A.; Buckup, P.A.; Figueiredo, J.L. \& Moura, R.L. Catálogo das espécies de peixes marinhos do Brasil. São Paulo, Museu de Zoologia da Universidade de São Paulo. 164 p., 2003.

Menni, R.C. \& Stehmann, M.F.W. Distribution, environment and biology of batoid fishes off Argentina, Uruguay and Brazil. A review. Revista del Museo Argentino de Ciencias Naturales Nueva Serie, v. 2, n. 1, p. 69-109, 2000. 
Nunan, G.W. \& Senna, M.L.V. Tubarões (Selachii) coletados pelo Navio Oceanográfico Thalassa sobre a plataforma externa e talude continental do Brasil entre $11^{\circ}$ e $22^{\circ} \mathrm{S}$, in Costa, P.A.S.; Olavo, G. \& Martins, A.S. (Eds.). Biodiversidade da fauna marinha profunda na costa central brasileira. Rio de Janeiro, Museu Nacional, p. 163-183, 2007.

Nunes, J.L.S.; Almeida, Z.D.D.S. \& Piorski, N.M. Raias capturadas pela pesca artesanal em águas rasas do Maranhão-Brasil. Arquivos de Ciências do Mar, v. 38, n. 1-2, p. 49-54, 2005.

Oliver, S.M.; Braccini, M.; Newman, S.J. \& Harvey, E.S. Global patterns in the bycatch of sharks and rays. Marine Policy, v. 54, p. 86-97, 2015.

Queiroz, B.J.; Neto, J.S.; Medeiros, R.S.; Nascimento, F.C.; Neto, M.A.D.A.F.; Faria, V.V. \& Rincon, G. Cartilaginous fishes (class Chondrichthyes) off Ceará state, Brazil, Western Equatorial Atlantic-an update. Arquivos de Ciências do Mar, v. 41, n. 2, p. 73-81, 2008.

Rezende, G.A.; Capitoli, R.R. \& Vooren, C.M. Dieta e morfologia da cabeça, boca e dentição de duas raias simpátricas, Myliobatis goodei e M. ridens (Batoidea: Myliobatiformes). Boletim do Museu de Biologia Mello Leitão, v. 37, n. 3, p. 255-270, 2015.

Rincon, G. Benthobatis kreffti. The IUCN Red List of Threatened Species 2004:e.T44577A10921737. 2004. Disponível em: http://dx.doi.org/10.2305/IUCN.UK.2004.RLTS.T44577A10921737. en. Acesso em: 12 jul. 2018.

Rincon, G.; Mazzoleni, R.C.; Palmeira, A.R.O. \& Lessa, R. Deep-Water Sharks, Rays, and Chimaeras of Brazil, in Chondrichthyes-Multidisciplinary Approach. InTech, p. 84-112, 2017.

Rosa, R.S. \& Gadig, O.B.F. Conhecimento da diversidade dos Chondrichthyes marinhos no Brasil: a contribuição de José Lima de Figueiredo. Arquivos de Zoologia (São Paulo), v. 45, n. especial, p. 89-104, 2014.

Rosa, R.S.; Furtado, M.; Snelson, F.; Piercy, A.; Grubbs, R.D.; Serena, F. \& Mancusi, C. Bathytoshia centroura. The IUCN Red List of Threatened Species 2016: e.T63152A104065289. 2016. Disponível em: http://dx.doi.org/10.2305/IUCN.UK.2016-3.RLTS.T63152A104065 289.en. Acesso em: 12 jul. 2018.

San Martín, J.M.; Stehmann, M.F.W. \& Kyne, P.M. Atlantoraja platana. The IUCN Red List of Threatened Species 2007: e.T63110A12608554. 2007. Disponível em: http://dx.doi. org/10.2305/IUCN.UK.2007.RLTS.T63110A12608554.en. Acesso em: 12 jul. 2018.

Santos, H.R.S.; Gomes, U.L. \& Charvet-Almeida, P. A new species of whiptail stingray of the genus Dasyatis Rafinesque, 1810 from the Southwestern Atlantic Ocean (Chondrichthyes: Myliobatiformes: Dasyatidae). Zootaxa, v. 492, n. 1, p. 1-12, 2004.

Silva, G.B.; Basílio, T.H.; Nascimento, F.C.P. \& Fonteles Filho, A.A. Tamanho na primeira maturidade sexual das raias Dasyatis guttata e Dasyatis americana, no litoral do estado do Ceará. Arquivos de Ciências do Mar, v. 40, n. 2, p. 14-18, 2007.

Soto, J.M.R.; Consulim, C.E.N. \& Souza Filho, M.D. First record of clark's fingerskate, Dactylobatus clarldi (Bigelow \& Schroeder, 1958) (Chondrichthyes, Rajidae), in the South Atlantic. Mare Magnum, v. 21, n. 1-2, p. 145-148, 2004.

Stehmann, M.F.W.; San Martín, J. \& Carvalho, M.R. Tetronarce puelcha. The IUCN Red List of Threatened Species 2006: e.T60135A12311366. 2006. Disponível em: http://dx.doi. org/10.2305/IUCN.UK.2006.RLTS.T60135A12311366.en. Acesso em: 12 jul. 2018. 
Tomás, A.R.G.; Gomes, U.L. \& Ferreira, B.P. Distribuição temporal dos elasmobrânquios na pesca de pequena escala de Barra de Guaratiba, Rio de Janeiro, Brasil. Boletim do Instituto de Pesca, v. 36, p. 317-324, 2010.

Worm, B.; Davis, B.; Kettemer, L.; Ward-Paige, C.A.; Chapman, D.; Heithaus, M.R.; Kessel, S.T. \& Gruber, S.H. Global catches, exploitation rates, and rebuilding options for sharks. Marine Policy, v. 40, p. 194-204, 2013.

Zar, J.H. Biostatistical Analysis: Pearson New International Edition. Pearson Higher Ed.,972 p., 2013. 